\title{
Non-collinear magnetic order coupled with magnetic glassy behaviour and enhancement of magnetocaloric effect under high pressure
}

\author{
Srikanta Goswami ${ }^{1}$, P. D. Babu ${ }^{1}$, R. Rawat ${ }^{2}$ \\ ${ }^{1} U G C-D A E$ Consortium for Scientific Research, Mumbai Centre, BARC Campus, Mumbai 400085, INDIA, \\ ${ }^{2} U G C-D A E$ Consortium for Scientific Research, University Campus, Khandwa Road, Indore- 452001, INDIA \\ srikantag@csr.res.in
}

Rare earth rich intermetallic compounds of the type $\mathrm{R}_{3} \mathrm{~T}(\mathrm{R}=$ rare earth, $\mathrm{T}=$ transition metal $)$ are found to be formed by very limited elements because of negative heat of formation and these compounds are revisited several times due to various novel properties emerging out of their crystal and magnetic structures. [1] $\mathrm{Tb}_{3} \mathrm{Co}$ is one such compound which orders below $\mathrm{T}_{\mathrm{N}}=84 \mathrm{~K}$ (Fig. 1) and goes through a first order magnetic transition of order-to-order type at $\sim 72 \mathrm{~K}$. Despite of several previous studies on this compound the reason behind the low temperature transition like feature was remain unexplained. It was revealed by our temperature dependent neutron diffraction study (Fig. 2) that the magnetic structure of this compound remains unaltered below $70 \mathrm{~K}$ to the lowest temperature except for some changes in intensity and moment values. The Rietveld refinement analysis to these data suggests that there is a drastic change in lattice parameters and volume around $40 \mathrm{~K}$ clearly suggesting a presence of strong spin-lattice coupling in this compound. Further, magnetic field dependent neutron diffraction data and the refinement analysis performed over the high angle part of these field dependent patterns confirms that there exists a change in strength of the spin-lattice coupling which is stronger at lower temperature and getting weaker at higher temperature. This is what responsible for the low temperature drop in ZFC in this compound. [2] Moreover, strong frequency dispersion in linear and nonlinear ac- susceptibility data and their various analyses confirm that $\mathrm{Tb}_{3} \mathrm{Co}$ exhibits magnetic glassy behaviour with magnetic glass temperature coincides with first order transition temperature at 72 K. [2]

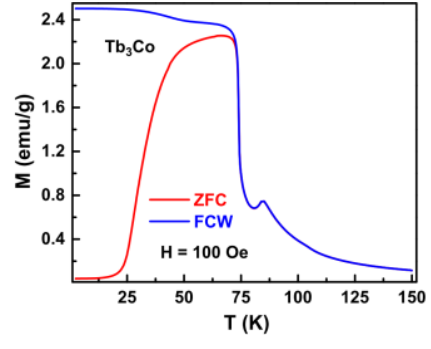

Figure 1. $\mathrm{ZFC}-\mathrm{FC}$ of $\mathrm{Tb}_{3} \mathrm{Co}$

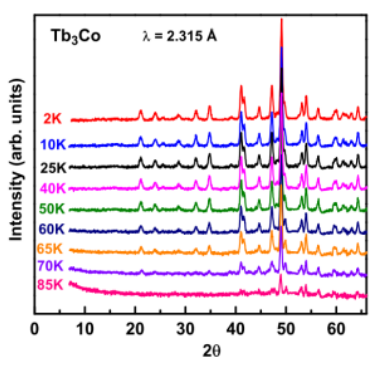

Figure 2. Temperature dependent ND pattern of $\mathrm{Tb}_{3} \mathrm{Co}$

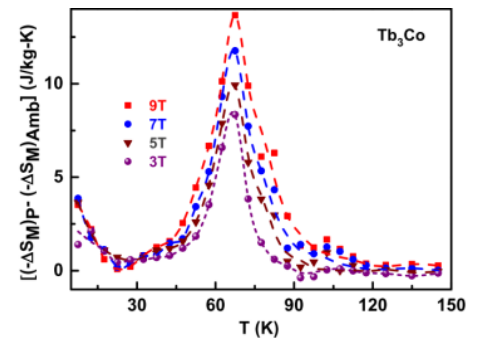

Figure 3. Enhancement of MCE under pressure in $\mathrm{Tb}_{3} \mathrm{Co}$

Further, various time dependent dc- magnetization measurements provide evidence for the fact that the magnetic glassy behaviour persists in this compound even up to $\mathrm{P} \sim 1 \mathrm{GPa}$ although the fitting to stretched exponential equation to the magnetic relaxation data infers that the glassy behaviour is weakened enough at $\mathrm{P}=0.69 \mathrm{GPa}$ and further increase in pressure has marginal effect on the glassy behaviour in this compound. [3] However, apart from shifting the transition temperatures to lower temperature $\left(\mathrm{T}_{\mathrm{N}}\right.$ by $6 \mathrm{~K}_{\text {, first order }}$ transition by $15 \mathrm{~K}$ and low temperature transition like feature by $3 \mathrm{~K}$ ) with increasing pressure up to $1.21 \mathrm{GPa}$, pressure is found to improve the magnetocaloric property of this compound (Fig. 3) to a large extent. The difference between $-\Delta \mathrm{S}_{\mathrm{M}}$ in presence of $\mathrm{P} \sim 1$ $\mathrm{GPa}$ and at ambient condition is plotted in Fig. 3 and it is exhibiting a strong peak thereby inferring an enhancement of $37 \%$ in magnetocaloric effect in $\mathrm{Tb}_{3} \mathrm{Co}$ at $\mathrm{P} \sim 1 \mathrm{GPa}$.

[1] Buschow, K. H. J. (1977) Rep. Prog. Phys. 401179.

[2] Goswami, S., Babu, P. D., Rawat, R. (2019). J. Phys. Condens. matter 31, 505802.

[3] Goswami, S., Babu, P. D., Rawat, R. (2019). J. Phys. Condens. matter 31, 505802.

Keywords: Rare earth rich intermetallic compounds; non-collinear AFM structure; magnetic glassiness; high pressure; magnetocaloric effect 